\title{
OVERVIEW OF ADOLESCENT MENSTRUAL PROBLEMS AND ITS RELATION TO BMI, EATING HABITS AND PHYSICAL ACTIVITY
}

\author{
Gauri Raghunath Shinde1, Manisha Laddad²
}

${ }^{1}$ Assistant Professor, Department of Obstetrics and Gynaecology, KIMSDU, Karad.

${ }^{2}$ Associate Professor, Department of Obstetrics and Gynaecology, KIMSDU, Karad.

\begin{abstract}
BACKGROUND

Purpose of this study is to overview adolescent menstrual problems and its relation to BMI, eating habits and physical activity.

\section{MATERIALS AND METHODS}

Present study carried out in Obstetrics \& Gynaecology Department, KIMSDU, Karad. In this study, 624 adolescent age group girls 11 to 19 yrs. who visited outpatient department were studied from Jan. 2013 to Dec. 2015. All these girls were asked for detailed history regarding age of menarche, menstrual pattern, any associated menstrual problems like dysmenorrh oea, pre-menstrual symptoms, history of passage of clots, menstrual irregularities. BMI of all these girls were calculated and categorised in to normal (18.5-24.99 $\left.\mathrm{kg} / \mathrm{m}^{2}\right)$, underweight (14-18.49 kg/m²), and overweight \& obese $\left(>25 \mathrm{~kg} / \mathrm{m}^{2}\right)$. All these girls were asked regarding their diet habits. Frequency of eating junk food (categorised into $<3$ days per week and $>3$ days per week). After all these detailed history collections, each girl underwent detailed general, per abdominal and local examination. All these girls underwent pelvic ultrasound to rule out major pelvic organic pathology. All these girls were further studied for the relation of dietary habits, BMI with the menstrual disorders. The girls diagnosed with menorrhagia were treated with Tab. Sylate $1 \mathrm{~g}$ tds for 5 days, haematinics or blood transfusion according to haemogram reports. Occasionally, few girls out of these required admission for acute events of menorrhagia \& treated accordingly. Statistical analysis was done to know the $\%$ of menstrual problems in these girls and these results were compared with different study results.
\end{abstract}

\section{RESULTS}

In present study, $14.42 \%$ girls were obese /overweight, $16.34 \%$ were underweight. $32.85 \%$ girls were with habit of eating junk food $>3$ days a week. 57.69\% girls giving history of physical activity $<3$ days a week. Dysmenorrhoea (66.34\%) was a commonest problem. Incidence of irregular cycles was $30.76 \%$, menorrhagia was $16.98 \%$, PMS was $48.71 \%$, abnormal duration of cycle in $14.42 \%$ and $16.34 \%$ girls were diagnosed as PCOD on USG. Overweight/obese and underweight girls had majority of menstrual problems (Dysmenorrhoea $76.66 \%$ vs. $40.19 \%$, irregular cycles $16.66 \%$ vs. $21.56 \%$, menorrhagia $16.66 \%$ vs. $21.56 \%$, PMS $41.11 \%$ vs. $53.92 \%$, abnormal duration of menses $13.33 \%$ vs. $14.70 \%$, PCOD $75.55 \%$ vs. 9.8\%). Maximum girls with dieting habits, less physical activity and habits of eating junk food were having multiple menstrual problems.

\section{CONCLUSION}

Changing lifestyle has increased incidences of bulimia nervosa, obesity, sedentary life style. All these things have an effect on developing adolescent girl. This is a vicious cycle of overeating/malnutrition adding to abnormal BMI which has an effect on physical activity of these girls which leads to menstrual problems. To overcome these problems in adolescent girls, awareness regarding physical activity, healthy eating habits, personal hygiene, counselling regarding diet to avoid increasing slim/plum body trends in adolescents should be done.

\section{KEYWORDS}

Adolescent Girl, Menstrual Problems, Adolescent Menstrual Problems.

HOW TO CITE THIS ARTICLE: Shinde GR, Laddad M.Overview of adolescent menstrual problems and its relation to BMI, eating habits and physical activity. J. Evolution Med. Dent. Sci. 2016;5(91):6757-6761, DOI: 10.14260/Jemds/2016/1526

\section{BACKGROUND}

Adolescence, the period between childhood and adulthood, is usually defined by the rapid onset of biological growth and development before or end of $2^{\text {nd }}$ decade of life. ${ }^{1}$ There are many factors which influence adolescent growth such as

Financial or Other, Competing Interest: None.

Submission 27-09-2016, Peer Review 31-10-2016,

Acceptance 07-11-2016, Published 12-11-2016.

Corresponding Author:

Dr. Gauri Raghunath Shinde,

C/o. Dr. Raghunath Shinde,

C/s. No. 1549, Opp. Swimming Tank,

Ganesh Nagar, Sangli-416416,

Maharashtra.

E-mail:drgauri8@gmail.com

DOI: $10.14260 /$ jemds/2016/1526

(c) (i) (3) $\Theta$ social, environmental, etc. Adolescent age group comprises of girls from 10 to 19 yrs. Menstrual disorders are common in this age group. Many of these problems are minor and can be resolved with education and reassurance about wide variation inherent in normal pubertal development. But some menstrual problems should be properly termed, investigated and treated. This article will discuss the current scenario of incidence of adolescent menstrual problems.

Over the past two decades, changing lifestyle, change in expectation of women in our society has presented adolescent girls with number of new challenges as well as new opportunities. Increasingly intense academic, social, athletic pressures create a stressful environment for these teenagers. This leads to eating disorders, anorexia nervosa, bulimia nervosa, increased incidences of PCOD and menstrual 
problems in adolescents. This article also focuses on effects of physical activity, nutrition, eating habits on menstrual cycle.

\section{Aim of the Study}

1. Overview of adolescent menstrual problems.

2. Relation of BMI, eating habits and physical activity to menstrual problems.

\section{MATERIALS AND METHODS}

Present study carried out in Obstetrics \& Gynaecology Department, KIMSDU, and Karad. In this study, 624 adolescent age group girls 11 to 19 yrs. who visited outpatient department were studied from Jan. 2013 to Dec. 2015. All these girls were asked for detailed history regarding age of menarche, menstrual pattern, any associated menstrual problems like dysmenorrhoea, premenstrual symptoms, history of passage of clots, menstrual irregularities. BMI of all these girls were calculated and categorised in to normal (18.5$\left.24.99 \mathrm{~kg} / \mathrm{m}^{2}\right)$, underweight ( $\left.14-18.49 \mathrm{~kg} / \mathrm{m}^{2}\right)$, and overweight \& obese $\left(>25 \mathrm{~kg} / \mathrm{m}^{2}\right)$. All these girls were asked regarding their diet habits. Frequency of eating junk food (categorised into $<3$ days per week and $>3$ days per week). After all these detailed history each girl underwent detailed general, per abdominal and local examination. All these girls underwent pelvic ultrasound to rule out major pelvic organic pathology. All these girls further studied for the relation of dietary habits, BMI with the menstrual disorders.

The girls diagnosed with menorrhagia were treated with Tb. Sylate $1 \mathrm{~g}$ tds for 5 days, haematinics or blood transfusion according to haemogram reports. Occasionally few girls out of these required admission for acute events of menorrhagia \& treated accordingly.

\section{Inclusion Criteria}

1. Girls who have attained menarche.

2. Girls from age group 11-19 yrs.

3. Girls with menstrual problems.

\section{Exclusion Criteria}

1. Girls already on hormonal line of treatment.

2. Girls with diagnosed cases of bleeding disorders.

3. Girls > than 19 yrs.

\section{RESULTS}

This study was carried out in Obstetrics \& Gynaecology Dept., KIMSDU, Karad. In this study, 624 adolescent girls fulfilling above-mentioned inclusion criteria visited to OB/GY OPD were studied. Out of these 624 girls, 22 girls required admission for acute episode menorrhagia. All these 22 girls were treated accordingly as mentioned in methodology.

In present study, 230 (36.85\%) girls were from age group 11-14 yrs. and 394 (63.14\%) from 15 to 19 yrs. age group.

\begin{tabular}{|c|c|c|}
\hline BMI & $\mathbf{n = 6 2 4}$ & $\mathbf{\%}$ \\
\hline Normal $\left(18.5-24.99 \mathrm{~kg} / \mathrm{m}^{2}\right)$ & 432 & 69.23 \\
\hline Underweight $\left(14-18.49 \mathrm{~kg} / \mathrm{m}^{2}\right)$ & 102 & 16.34 \\
\hline Overweight/obese $\left(>25 \mathrm{~kg} / \mathrm{m}^{2}\right)$ & 90 & 14.42 \\
\hline \multicolumn{2}{|c|}{ Table 1. Categorised According to BMI } \\
\hline
\end{tabular}

In present study, 506 (81.08\%) girls were from Urban residential area and 118 (18.91\%) were from Rural.

In present study, 175 (28.04\%) girls had habits of dieting \& remaining 449 (71.95\%) had no habits of dieting.
In present study, out of 624 girls, 419 (67.14\%) girls were having habit of eating junk food $<3$ days a week \& 205 $(32.85 \%)$ girls were having habit of junk food $>3$ days a week.

In present study, 360 (57.69\%) girls were having a habit of exercise $<3$ days a week and remaining 264 (42.30\%) girls were having a habit of daily exercise.

\begin{tabular}{|c|c|c|}
\hline Menstrual Problems in Adolescent girls & & $\mathbf{\%}$ \\
\hline Dysmenorrhoea & 414 & 66.34 \\
\hline Irregular cycles & 192 & 30.76 \\
\hline Menorrhagia & 106 & 16.98 \\
\hline Premenstrual symptoms (PMS) & 304 & 48.71 \\
\hline Abnormal duration of menses & 90 & 14.42 \\
\hline $\begin{array}{c}\text { Diagnosis of polycystic ovarian } \\
\text { disease(PCOD) }\end{array}$ & 102 & 16.34 \\
\hline \multicolumn{2}{|c|}{ Table 2. Menstrual Problems in Adolescent Girls } \\
\hline
\end{tabular}

In present study, commonest menstrual problem was dysmenorrhoeal (66.34\%), incidence of menorrhagia was $16.98 \%$. Many of the girls were having combination of menstrual problems mentioned above.

\begin{tabular}{|c|c|c|c|c|c|c|}
\hline$\sum_{n}$ & 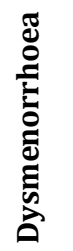 & $\delta^{0}$ & 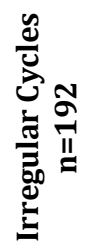 & $\delta^{0}$ & 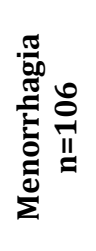 & de \\
\hline Normal & 304 & 70.37 & 155 & 35.87 & 69 & 15.97 \\
\hline $\begin{array}{l}\text { Under- } \\
\text { weight }\end{array}$ & 41 & 40.19 & 22 & 21.56 & 22 & 21.56 \\
\hline $\begin{array}{c}\text { Over- } \\
\text { weight/ } \\
\text { obese }\end{array}$ & 69 & 76.66 & 15 & 16.66 & 15 & 16.66 \\
\hline
\end{tabular}

In present study, incidence of dysmenorrhoea (76.66) was highest in overweight girls while irregular cycles (35.87\%) were more in normal BMI girls and menorrhagia in underweight girls.

\begin{tabular}{|c|c|c|c|c|c|c|}
\hline$\sum_{M}$ & 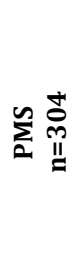 & $\partial^{0}$ & 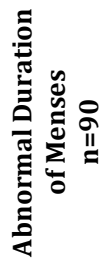 & $0^{\circ}$ & 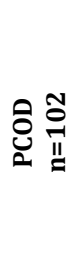 & $0^{\circ}$ \\
\hline Normal & 212 & 49.07 & 63 & 14.58 & 24 & 5.55 \\
\hline $\begin{array}{l}\text { Under- } \\
\text { weight }\end{array}$ & 55 & 53.92 & 15 & 14.70 & 10 & 9.8 \\
\hline $\begin{array}{l}\text { Over- } \\
\text { weight } \\
\text { /obese }\end{array}$ & 37 & 41.11 & 12 & 13.33 & 68 & 75.55 \\
\hline
\end{tabular}

Incidence of PMS (53.92\%) was highest in underweight girls, PCOD (75.55\%) highest in overweight girls.

Adolescent menstrual problems were mainly due to premature HPO (Hypothalamo-pituitary- Ovarian Axis), 
added to it BMI also play a role in these menstrual problems in

adolescent girl as shown in above tables 3-4.

\begin{tabular}{|c|c|c|c|c|c|c|}
\hline Dieting Habits & $\begin{array}{c}\text { Dysmenorrhoea } \\
\mathbf{n = 4 1 4}\end{array}$ & $\mathbf{\%}$ & $\begin{array}{c}\text { Irregular Cycles } \\
\mathbf{n = 1 9 2}\end{array}$ & $\mathbf{\%}$ & $\begin{array}{c}\text { Menorrhagia } \\
\mathbf{n = 1 0 6}\end{array}$ & \begin{tabular}{c} 
\% \\
\hline Yes
\end{tabular} \\
\hline No & 136 & 71.71 & 99 & 56.57 & 35 & 20 \\
\hline \multicolumn{7}{|c|}{ Table 5. Relation of Dieting Habits to Adolescent Menstrual Problems } \\
\hline
\end{tabular}

Dysmenorrhoea (71.71\%), irregular cycles (56.57\%), menorrhagia (20\%) were more in girls with dieting habits.

\begin{tabular}{|c|c|c|c|c|c|c|}
\hline $\begin{array}{c}\text { Dieting } \\
\text { Habits }\end{array}$ & $\begin{array}{c}\text { PMS } \\
\mathbf{n = 3 0 4}\end{array}$ & $\mathbf{\%}$ & $\begin{array}{c}\text { Abnormal Duration } \\
\text { of Menses } \\
\mathbf{n = 9 0}\end{array}$ & $\mathbf{\%}$ & $\begin{array}{c}\text { PCOD } \\
\mathbf{n = 1 0 2}\end{array}$ & $\mathbf{\%}$ \\
\hline Yes & 115 & 65.71 & 23 & 13.14 & 13 & 7.42 \\
\hline No & 189 & 42.90 & 67 & 14.92 & 89 & 19.82 \\
\hline \multicolumn{7}{|c|}{ Table 6. Relation of Dieting Habits to Adolescent Menstrual Problems } \\
\hline
\end{tabular}

PMS (65.71\%) was more common in girls with dieting habits while PCOD (19.82\%) was more in in girls without dieting habits.

\begin{tabular}{|c|c|c|c|c|c|c|}
\hline $\begin{array}{c}\text { Frequency of } \\
\text { Eating Junk } \\
\backslash \text { Food }\end{array}$ & $\begin{array}{c}\text { Dysmenorrhoea } \\
n=414\end{array}$ & $\%$ & $\begin{array}{c}\text { Irregular } \\
\text { Cycles } \\
n=192\end{array}$ & $\%$ & $\begin{array}{c}\text { Menorrhagia } \\
n=106\end{array}$ & $\%$ \\
\hline$<3$ days a week & 289 & 68.97 & 157 & 37.47 & 65 & 15.51 \\
\hline$>3$ days a week & 125 & 60.97 & 35 & 17.07 & 25 & 12.19 \\
\hline
\end{tabular}

Dysmenorrhoea (68.97\%), menorrhagia (15.51\%) were more in girls with habit of eating junk food $<3$ days a week.

\begin{tabular}{|c|c|c|c|c|c|c|}
\hline $\begin{array}{c}\text { Frequency of Eating } \\
\text { Junk Food }\end{array}$ & $\begin{array}{c}\text { PMS } \\
\mathbf{n = 3 0 4}\end{array}$ & $\mathbf{\%}$ & $\begin{array}{c}\text { Abnormal Duration } \\
\text { of Menses } \\
\mathbf{n = 9 0}\end{array}$ & $\mathbf{\%}$ & $\begin{array}{c}\text { PCOD } \\
\mathbf{n = 1 0 2}\end{array}$ & $\mathbf{\%}$ \\
\hline$<3$ days a week & 202 & 48.21 & 66 & 15.75 & 42 & 10.02 \\
\hline$>3$ days a week & 102 & 49.75 & 24 & 11.70 & 60 & 29.26 \\
\hline \multicolumn{6}{|r}{} \\
\hline
\end{tabular}

PMS (49.75\%), PCOD (29.26\%) were more in girls who were frequently (>3 days a week) eating junk food.

\begin{tabular}{|c|c|c|c|c|c|c|}
\hline $\begin{array}{c}\text { Frequency of } \\
\text { Physical } \\
\text { Exercise }\end{array}$ & $\begin{array}{c}\text { Dysmenorrhoea } \\
n=414\end{array}$ & $\%$ & $\begin{array}{c}\text { Irregular } \\
\text { Cycles } \\
\text { n=192 }\end{array}$ & $\%$ & $\begin{array}{c}\text { Menorrhagia } \\
n=106\end{array}$ & $\%$ \\
\hline$<3$ days a week & 298 & 82.77 & 157 & 43.61 & 70 & 19.44 \\
\hline$>3$ days a week & 116 & 43.93 & 35 & 13.25 & 36 & 13.63 \\
\hline
\end{tabular}

Girls with less frequency of physical exercise had a more incidence of dysmenorrhoeal (82.77\%), irregular cycle (43.61\%) and menorrhagia (19.44\%).

\begin{tabular}{|c|c|c|c|c|c|c|}
\hline $\begin{array}{c}\text { Frequency of Physical } \\
\text { Exercise }\end{array}$ & $\begin{array}{c}\text { PMS } \\
\text { n=304 }\end{array}$ & $\%$ & $\begin{array}{c}\text { Abnormal } \\
\text { Duration of } \\
\text { Menses } \\
\mathbf{n}=90\end{array}$ & $\%$ & $\begin{array}{l}\text { PCOD } \\
\mathrm{n}=102\end{array}$ & $\%$ \\
\hline$<3$ days a week & 157 & 43.61 & 51 & 14.16 & 69 & 19.16 \\
\hline$>3$ days a week & 147 & 55.68 & 39 & 14.77 & 33 & 12.5 \\
\hline
\end{tabular}

\section{DISCUSSION}

Purpose of present study was to take an overview of adolescent menstrual problems and their relation to physical exercise, BMI and habit of eating junk food. In present study, mean age was $14.20 \mathrm{yrs}$., mean age of menarche was $12.5 \mathrm{yrs}$. Majority of girls, $69.23 \%$ were with normal BMI, $81.08 \%$ were from urban area, $71.95 \%$ girls were with no dieting habits, $32.85 \%$ girls with habit of eating junk food $>3$ days a week and only $42.30 \%$ girls with habit of physical exercise $>3$ days a week. 


\begin{tabular}{|c|c|c|c|c|c|c|c|c|}
\hline $\begin{array}{c}\text { Adolescent } \\
\text { Menstrual } \\
\text { Problems }\end{array}$ & $\begin{array}{c}\text { Present } \\
\text { Study }\end{array}$ & $\begin{array}{c}\text { Nag } \\
\text { et al }{ }^{2}\end{array}$ & $\begin{array}{c}\text { George \& } \\
\text { Bhadhuri } \\
{\text { et } \mathbf{a l}^{3}}^{3}\end{array}$ & $\begin{array}{c}\text { Anil } \\
\text { Agarwal } \\
\text { et al4 }\end{array}$ & $\begin{array}{l}\text { Saira } \\
\text { Dars } \\
\text { et al5 }\end{array}$ & $\begin{array}{c}\text { Franco } \\
\text { Rigon } \\
\text { et al6 }\end{array}$ & $\begin{array}{c}\text { Rupa } \\
\text { Vani } \\
\text { et } \text { al }^{7}\end{array}$ & $\begin{array}{c}\text { Rama Ravi } \\
\text { et al }{ }^{8}\end{array}$ \\
\hline Dysmenorrhoea & $66.34 \%$ & $33.5 \%$ & $87.87 \%$ & $79.67 \%$ & $62 \%$ & $6.2 \%$ & $73 \%$ & $72.6 \%$ \\
\hline $\begin{array}{l}\text { Irregular length } \\
\text { of cycles }\end{array}$ & $30.76 \%$ & - & - & - & $24 \%$ & $9 \%$ & $17 \%$ & $31.7 \%$ \\
\hline Menorrhagia & $16.98 \%$ & - & - & - & $17 \%$ & $19 \%$ & $37 \%$ & $45.7 \%$ \\
\hline $\begin{array}{c}\text { Abnormal } \\
\text { duration of } \\
\text { menses }\end{array}$ & $14.42 \%$ & - & - & - & $29 \%$ & - & $10 \%$ & - \\
\hline PMS & $48.71 \%$ & - & - & - & $38 \%$ & - & $51 \%$ & - \\
\hline PCOD & $16.34 \%$ & - & - & - & - & - & - & - \\
\hline \multicolumn{9}{|c|}{ Table 11. Incidence of Adolescent Menstrual Problems in Different Studie } \\
\hline
\end{tabular}

Present study observations were nearly correlating to Saira Dars et al5, Rupa Vani etal7, and Rama Ravi et al ${ }^{8}$ Difference in observations in present study and Nag et al ${ }^{3}$,

George et al3 , Anil Agarwal et al ${ }^{4}$, Franco Rigon et al ${ }^{8}$ may be because of difference in socioeconomic status of population included in study and number of girls involved in study.

\begin{tabular}{|c|c|c|c|c|c|c|}
\hline $\begin{array}{c}\text { Study } \\
\text { Population }\end{array}$ & $\begin{array}{c}\text { Present } \\
\text { Study }\end{array}$ & $\begin{array}{c}\text { Anil } \\
\text { Agarwal } \\
\text { et al } \mathbf{l}^{4}\end{array}$ & $\begin{array}{c}\text { Saira Dars } \\
\text { et al5 }\end{array}$ & $\begin{array}{c}\text { Franco } \\
\text { Rigon et al6 }\end{array}$ & $\begin{array}{c}\text { Rupa } \\
\text { Vani et al7 }\end{array}$ & $\begin{array}{c}\text { Rama Ravi } \\
\text { et al8 }\end{array}$ \\
\hline BMI & 624 & 970 & 401 & 4892 & 853 & 350 \\
\hline $\begin{array}{c}\text { Normal } \\
\left(18.5-24.99 \mathrm{~kg} / \mathrm{m}^{2}\right)\end{array}$ & $69.23 \%$ & - & $69 \%$ & - & $52.2 \%$ & - \\
\hline $\begin{array}{c}\text { Under-weight } \\
\left(14-18.49 \mathrm{~kg} / \mathrm{m}^{2}\right)\end{array}$ & $16.34 \%$ & - & $27 \%$ & - & $34.5 \%$ & - \\
\hline $\begin{array}{c}\text { Overweight } / \mathrm{obese} \\
\left(>25 \mathrm{~kg} / \mathrm{m}^{2}\right)\end{array}$ & $14.42 \%$ & - & $04 \%$ & - & $13.3 \%$ & - \\
\hline
\end{tabular}

There was difference in number of study population in present study and above-mentioned studies. In spite of these differences; population with the normal, underweight, overweight BMI nearly correlated with Saira Dars et al ${ }^{5}$ and with Rupa Vani et al ${ }^{7}$ study.

\begin{tabular}{|c|c|c|c|c|c|}
\hline \multicolumn{6}{|c|}{ Present Study } \\
\hline & $\begin{array}{c}\text { Dysmenorrhoea } \\
\text { (\%) }\end{array}$ & $\begin{array}{c}\text { Irregular Cycles } \\
(\%)\end{array}$ & $\begin{array}{c}\text { Menorrhagia } \\
\text { (\%) }\end{array}$ & $\begin{array}{l}\text { PMS } \\
(\%)\end{array}$ & $\begin{array}{c}\text { Abnormal } \\
\text { Duration of } \\
\text { Menses } \\
\text { (\%) }\end{array}$ \\
\hline \multicolumn{6}{|l|}{ BMI } \\
\hline Normal $\left(18.5-24.99 \mathrm{~kg} / \mathrm{m}^{2}\right)$ & 70.37 & 35.87 & 15.97 & 49.07 & 14.58 \\
\hline Underweight(14 -18.49 kg/m²) & 40.19 & 21.56 & 21.56 & 53.92 & 14.70 \\
\hline Overweight /obese (>25 kg/m²) & 76.66 & 16.66 & 16.66 & 41.11 & 13.33 \\
\hline \multicolumn{6}{|l|}{ Dieting habits } \\
\hline Yes & 71.71 & 56.57 & 20 & 65.71 & 13.14 \\
\hline No & 61.97 & 20.71 & 15.81 & 42.90 & 14.92 \\
\hline \multicolumn{6}{|l|}{ Frequency of eating junk food } \\
\hline$<3$ days a week & 68.97 & 37.47 & 15.51 & 48.21 & 15.75 \\
\hline$>3$ days a week & 60.97 & 17.07 & 12.19 & 49.75 & 11.70 \\
\hline \multicolumn{6}{|l|}{ Frequency of physical } \\
\hline$<3$ days a week & 82.77 & 43.61 & 19.44 & 43.61 & 14.16 \\
\hline$>3$ days a week & 43.93 & 13.25 & 13.63 & 55.68 & 14.77 \\
\hline Table 13. Association of $\mathrm{BMI}$ & $\begin{array}{r}\text { ing Habits, Junk } \\
\text { Present Study }\end{array}$ & $\begin{array}{l}\text { eating, Physical } \\
\text { Study by Rupa V }\end{array}$ & $\begin{array}{l}\text { ise to Adoles } \\
a l^{7}\end{array}$ & Iens & oblems in \\
\hline
\end{tabular}




\begin{tabular}{|c|c|c|c|c|c|}
\hline \multicolumn{6}{|c|}{ Rupa Vani et al7 } \\
\hline & $\begin{array}{c}\text { Dysmenorrhoea } \\
\text { (\%) }\end{array}$ & $\begin{array}{c}\text { Irregular Cycles } \\
\text { (\%) }\end{array}$ & $\begin{array}{c}\text { Menorrhagia } \\
\text { (\%) }\end{array}$ & $\begin{array}{l}\text { PMS } \\
(\%)\end{array}$ & $\begin{array}{c}\text { Abnormal } \\
\text { Duration } \\
\text { of Menses } \\
(\%)\end{array}$ \\
\hline \multicolumn{6}{|l|}{ BMI } \\
\hline Normal $\left(18.5-24.99 \mathrm{~kg} / \mathrm{m}^{2}\right)$ & 69.4 & 17.8 & 33.5 & 52.8 & 24.3 \\
\hline $\begin{array}{c}\text { Underweight } \\
\left(14-18.49 \mathrm{~kg} / \mathrm{m}^{2}\right)\end{array}$ & 76.5 & 15.3 & 40.5 & 45.9 & 23.1 \\
\hline Overweight /obese (>25 kg/m²) & 76.3 & 21.9 & 41.2 & 58.8 & 27.2 \\
\hline \multicolumn{6}{|l|}{ Dieting habits } \\
\hline Yes & 78.1 & 18.8 & 43.4 & 67.2 & 26.2 \\
\hline No & 70.5 & 16. & 34.2 & 44.4 & 23.5 \\
\hline \multicolumn{6}{|l|}{ Frequency of eating junk food } \\
\hline$<3$ days a week & 71.4 & 18.6 & 36 & 47.7 & 24.7 \\
\hline$>3$ days a week & 75.6 & 15.3 & 38.7 & 58.2 & 23.3 \\
\hline \multicolumn{6}{|l|}{ Frequency of physical } \\
\hline$<3$ days a week & 72 & 18.6 & 37.7 & 48.4 & 25.7 \\
\hline$>3$ days a week & 74.8 & 14.6 & 35 & 56.4 & 20.7 \\
\hline \multicolumn{6}{|c|}{ Table 14} \\
\hline
\end{tabular}

Observations in association of BMI, Dieting habits, eating Junk food, physical exercise to Adolescent menstrual problems in present study and study by Rupa Vani et al ${ }^{7}$ were nearly correlating. This suggests that changing lifestyle, eating habits, frequency of physical activity \& BMI have an effect on adolescent menstrual cycles.

In present study, incidence of diagnosed PCOD on USG was high in overweight \& obese BMI girls (75.75\%). 19.82\% of girls without dieting history were diagnosed as PCOD on USG. $29.26 \%$ girls eating junk food $>3$ days a week, diagnosed as PCOD on USG. $19.16 \%$ of girls who were doing physical activity $<3$ days a week were diagnosed as PCOD on USG. All these girls diagnosed as PCOD were having combination of menstrual problems.

\section{CONCLUSION}

Present study is an overview of adolescent menstrual problems and its relation to BMI, eating habits, physical activity. Menstrual problems are common in adolescent girls because of premature HPO Axis physiologically. But changing lifestyle has increased incidences of bulimia nervosa, anorexia nervosa, obesity, sedentary life style. All these things have an effect on developing adolescent girl. This is a vicious cycle of over eating/malnutrition adding do abnormal BMI which has an effect on physical activity of these girls which leads to menstrual problems. To overcome these problems in adolescent girls, awareness regarding physical activity, healthy eating habits, personal hygiene and counselling regarding diet to avoid increasing slim/plum body trends in adolescent should be done.

\section{REFERENCES}

1. Nagrath A, Kumar P, Ruprai RK. Adolescent gynecology. Chapter 6. In: Arulkumar S, Sivanesaratnam V, Chatterjee A, et al. (eds) Essentials of gynecology. $1^{\text {st }}$ edn. Jaypee brothers medical publishers (P) Ltd 2005:43.

2. Nag RM. Adolescent in India. Calcutta: Medical Allied Agency 1982:18-26.

3. George A, Bhaduri A. Dysmenorrhea among adolescent girls - symptoms experienced during menstruation. Health Promotion Educ 2002;17:4.

4. Agarwal AK, Agarwal A. A study of dysmenorrhea during menstruation in adolescent girls. Indian J Community Med 2010;35(1):159-64.

5. Dars S, Sayed K, Yousufzai Z. Relationship of menstrual irregularities to BMI and nutritional status in adolescent girls. Pak J Med Sci 2014;30(1):141-4.

6. Rigon F, De Sanctis V, Bernasconi S, et al. Menstrual pattern and menstrual disorders among adolescents: an update of the Italian data. Ital J Pediatr 2012;38:38.

7. Vani RK, Veena KS, Subitha L, et al. Menstrual abnormalities in school going girls-are they related to dietary and exercise pattern? Journal List J Clin Diagn Resv 2013;7(11):2537-40.

8. Ravi R, Shah P, Palani G, et al. Prevalence of menstrual problems among adolescent school girls in rural Tamil Nadu. Journal of Pediatric \& Adolescent Gynecology 2016;29(6):571-6. 